\title{
Numerical method of active thermography for the reconstruction of back wall geometry
}

\author{
by Regina Richter ${ }^{*}$, Christiane Maierhofer ${ }^{*}$, Marc Kreutzbruck $^{*}$
}

*BAM Federal Institute for Materials Research and Testing, Division 8.4, Unter den Eichen 87, 12205 Berlin, Germany, regina.richter@bam.de

\section{Abstract}

The paper presents a numerical method to detect and characterise defects and inhomogeneities by means of active thermography. The objective is to determine the wall thickness of structure elements with an inaccessible back wall, e.g. of pipes or containers. As test specimens we used $2 \mathrm{~cm}$ thick PVC samples with flat bottom holes in the back wall. Flash lamps provided the heating. The inversion method is the Levenberg-Marquardt method, which starts by a rough first guess of the back wall geometry using the echo defect shape method, and then the PVC thickness of specimen profiles was calculated. We validated the result by comparing the measurement data to simulated data for selected geometries.

\section{Introduction}

Active thermography like impulse-thermography is popular because it allows inspecting the structure very quickly. The application of flash lamps is common: This technique consists of a short light impulse radiated from flash lamps and heating up the structure element. By analysing the surface temperature we can detect inhomogeneities: the surface temperature decays as long as the heat can flow into the interior. To obtain not only qualitative, but also quantitative data, an overview of methods for data analysis of flash excitation is given in Maldague [1], Sun [2] and Omar [3].

In this paper, we discuss in detail the performance of an iterative reconstruction algorithm, depending on the number of iteration steps and on the shape characteristics. The applied algorithm is an optimised version of a classical method for inverse problems. Why do we have an inverse problem inhere? We measure the effect of a physical process, i.e. the devolution of the intensity of thermal radiation of the surface after a flash excitation, but we are interested in the cause of this physical process: The exact geometry of the test specimen. From this point of view, inverse problems offer the perfect tool kit for tackling this challenge.

In the work presented herein, we reconstructed back wall geometries using an inversion method based on the minimisation of the linearised problem within a trust region: the Levenberg-Marquardt method [4].

The advantages of the Levenberg-Marquardt method are

1) this method uses the information of the whole time interval, not only the temperature for a certain time

2) lateral heat diffusion effects are considered

3) the convergence behaviour is well understood, classified and trustworthy (in a theoretical and general sense)

4) this method has proven its reliable applicability in other areas and is very popular, like e.g. in geophysics [5]

\section{Numerical methods for reconstructing the wall thickness}

For yielding the quantitative information of the wall thickness, we applied an inversion method. Figure 1 presents the general principle of the complete procedure.

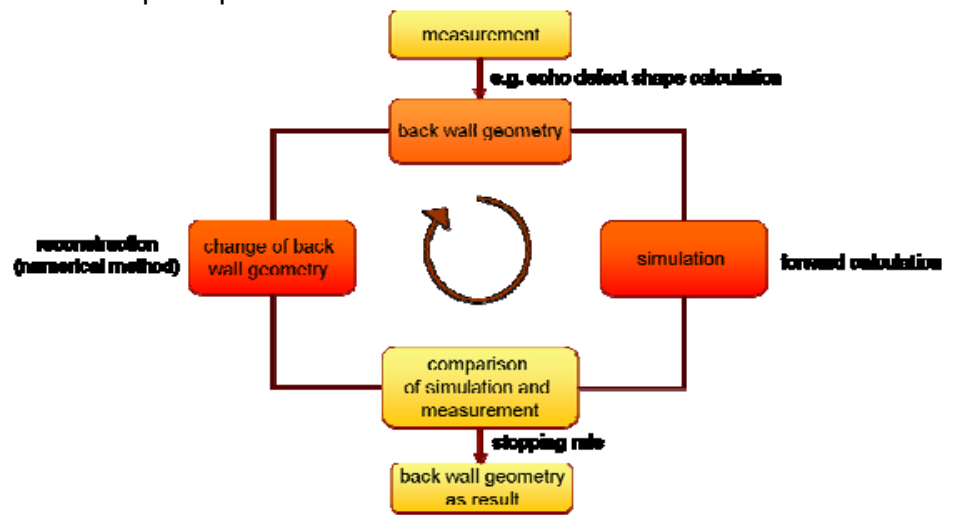

Figure 1: The complete inversion procedure 
First, we are performing measurements yielding experimental data, from which we are calculating an initial back wall geometry using the echo defect shape method [6]. Second, we simulate the temperature devolution (forward calculation) for the assumed structure profile by using the FEM based software COMSOL. Third, by comparing the results of simulation with the experimental data, we change the back wall geometry by an inversion method (reconstruction using Levenberg-Marquardt method). The forward calculation and the reconstruction are done iteratively. Finally, the circle is stopped after 40 iterations, as the geometry changes stagnated. Within the algorithm, the maximum possible sample thickness was fixed (first a priori knowledge).

\subsection{Initial geometry using the echo defect shape method}

To obtain a rough idea of the back wall geometry, we applied the echo defect shape method, for details see Lugin [6]. To derive this depth formula, the analytical 1D solution for the heat equation is calculated for a specimen with semi-infinite thickness which is exposed to a Dirac delta pulse heating on the front. The 1D thermal wave model declares, that for a specimen with given finite thickness $z$, reflection of the thermal wave on the back occurs and leads to a temperature increase $T_{\text {defect }}(t)-T_{\text {reference }}(t)$ at the front which is equal to the temperature of the semi-infinite specimen at the depth $2 z$. Assuming $T_{\text {reference }}(t)$ is equal to the temperature of the semi-infinite specimen at the front and considering the relative temperature increase

$$
C_{\text {rel }}(t)=\frac{T_{\text {defect }}(t)-T_{\text {reference }}(t)}{T_{\text {reference }}(t)}
$$

with respect to a defect free reference area, the specimen thickness can be evaluated by

$$
Z=\sqrt{a \cdot t \cdot\left(-\ln \left(C_{r e l}(t)\right)\right.}
$$

The variable $T$ denotes the temperature difference to room temperature, $t$ is the time elapsed after the delta pulse heating, and $a$ is the thermal diffusivity of the material. Eq. (2) is evaluated for the time $t$, for which the relative contrast reaches a chosen benchmark value. We predisposed the quite small benchmark $C_{r e l}=0.035$, as the relative contrast of even deep defects should reach this benchmark, although the noise of the experimental data spoiled certain reference areas.

To conclude, the echo defect shape method is here applied as Eq. (3): If $t$ is the first time, for which $C_{r e l}=0.035$ is valid, then we can evaluate the thickness with

$$
z=\sqrt{a \cdot t \cdot(-\ln (0.035))}
$$

As the echo defect shape covers only the effects of 1D heat flow, we enhanced the back wall geometry of interesting profiles with an iterative method (see next chapter). For these calculations, it was helpful to notice, that the specimen was best reconstructed where the specimen was thinnest compared to its close surroundings (second a priori knowledge).

For quantifying the general accuracy of the calculated geometry $z$ of a profile, we expressed the error on average by the arithmetic mean:

$$
\text { error }=\frac{\sum_{i=1}^{n}\left|r^{i}-z^{i}\right|}{n},
$$

where $r^{i}$ is the thickness of the real geometry (at the $i$-th front point), $z^{i}$ the calculated geometry and $n$ the number of surface points along a measurement line.

\subsection{Reconstruction using the Levenberg-Marquardt method}


To describe the Levenberg-Marquardt method easily, we explain it on a simple defect. First, we need to define here the forward problem. Let us consider a $15 \mathrm{~cm}$ long and $15 \mathrm{~cm}$ wide 3D specimen with varying thickness between 2 and $0.3 \mathrm{~cm}$ with a notch shaped defect, see figure 2 .

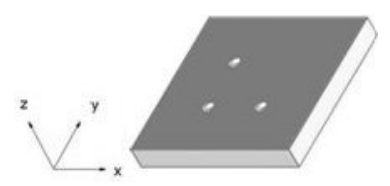

Figure 2. The flat-bottom hole sample in $3 D$

Our goal in this work is a 2D reconstruction, i.e. the initial 3D geometry was sliced at its defect centres. Thus, let us consider a $15 \mathrm{~cm}$ long specimen profile with varying thickness between 2 and $0.3 \mathrm{~cm}$, see figure 3 . We describe the back wall by a finite number of points and interpolate the thickness linearly in between. To show easily the principle, we explain here the example for $15+1$ equidistant sampling points on the $15 \mathrm{~cm}$ long front, i.e. we have sampling points with $1 \mathrm{~cm}$ distances.

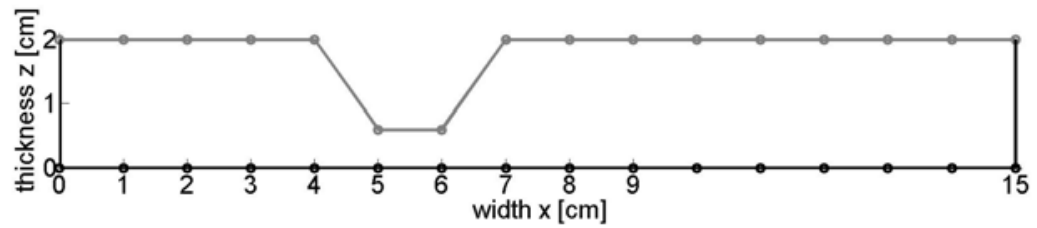

Figure 3. Example of a back wall geometry (grey circles) given by the thickness at 15+1 sampling points at the front (black circles)

We were able to measure or simulate the temperature devolution after flash heating for a given specimen with a given back wall, e.g. $Z_{\text {real }}=(2,2,2,2,2,1.5,0.9,0.3,0.9,1.5,2,2,2,2,2,2)$ as shown in figure 3 . The temperature devolution of the experimental data had a discrete formulation, as we measured with 475 pixels (FPA IR camera) on the profile front and on discrete points in time. I.e. for each second of the time interval $[0,600] \mathrm{s}$, we recorded the spatial temperature devolution on the profile front. We align the temperature devolution $T$ in a vector with $(475 \times 601)$ entries: first the temporal temperature devolution of the first pixel, then of the second pixel, and so on, see figure 4.

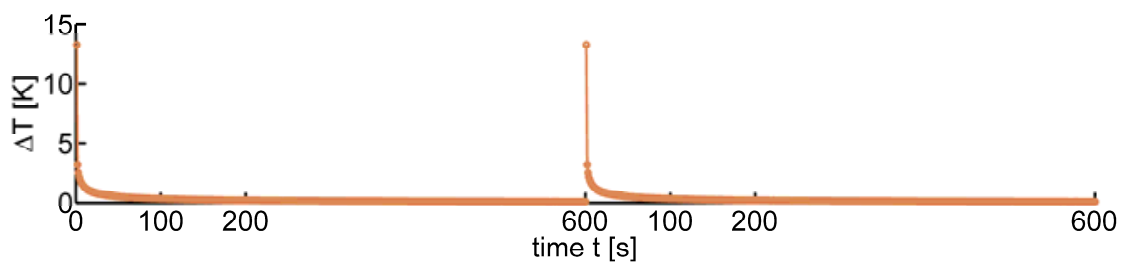

Figure 4. $T$ begins with the temperature for the time interval $[0,600] \mathrm{s}$ for the first pixel, and it continues with the temperature for the same time interval for the second pixel (and so on). $T$ denotes the measured spatial and temporal temperature devolution (as difference to room temperature).

The vector function $F$ labels the relation of a given back wall $Z_{\text {real }}$ to the respective temperature devolution $T$. Thus, $F\left(z_{\text {real }}\right)=T=(13.30,3.24,2.54, \ldots, 13.29,3.23,2.54, \ldots)$, all vectors are column vectors. Using either simulated or measured $T$ values, in both cases, $F$ describes the solvable forward problem. 
Reversing the forward problem means reconstruction: How can you reconstruct $Z_{\text {real }}$, when $T$ is given by the experiment? The inverse function $F^{-1}$ for $F^{-1}(T)=z_{\text {real }}$ is unknown, thus, a linearisation of $F$ was considered, leading to the iterative Newton type method ${ }^{1}$ :

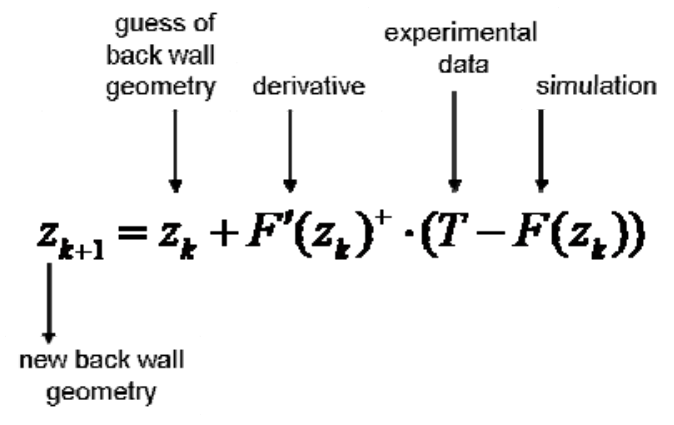

The initial geometry $Z_{1}$, was computed by the echo defect shape method Eq. (3) on the experimental data, and was meant to be enhanced in each iteration step of the Levenberg-Marquardt method, such that the $k$-th geometry $Z_{k}$ converges to the real back wall geometry $Z_{\text {real }}$. In each iteration step $k$, the comparison of simulated and measured temperature data $\left(T-F\left(z_{k}\right)\right)$ yields a correction of the back wall. The deviation $\left(T-F\left(z_{k}\right)\right)$ is weighted by the derivative $F^{\prime}\left(z_{k}\right)$, which is marked by a superscript + , meaning it is pseudo-inverted (analogue to the reciprocal of a real number).

In a nutshell, the derivative $F^{\prime}\left(z_{k}\right)$ describes the change of the thermal behaviour for tiny changes in the $k$-th geometry $Z_{k}$. We have performed the time consuming calculation of the derivative only for certain iteration steps, e.g. the initial geometry $z_{1}$. Going more into details, the derivative $F^{\prime}\left(z_{1}\right)$, at the geometry $z_{1}=\left(z^{1}{ }_{1}, z^{2}{ }_{1}, z^{3}{ }_{1}, \ldots, z^{16}{ }_{1}\right)$, is a non-quadratic matrix with the entry

$$
F^{\prime}\left(z_{1}\right)_{i j}=\frac{F_{i}\left(z^{1}{ }_{1}, z^{2}{ }_{1}, z^{3}{ }_{1}, \ldots, z^{j}{ }_{1}-0.01, \ldots, z^{16}{ }_{1}\right)-F_{i}\left(z_{1}\right)}{-0.01}
$$

in its $i$-th row and $j$-th column. The low index of $z$ designates the iteration step, the high index designates the relating front point number. The $i$-th information $F_{i}$ of the temperature devolution is the temperature at one of the 475 front points for a certain point in time. For example, $F_{1}$ is the temperature at $x=0 \mathrm{~cm}$ and $t=0 \mathrm{~s}, F_{2}$ is the temperature at $x=0 \mathrm{~cm}$ and $t=1 \mathrm{~s}$, and $F_{602}$ is the temperature at $x=1 \mathrm{~cm}$ and $t=0 \mathrm{~s}$. As $F_{i}\left(z_{1}\right)$ denotes the temperature for the specimen with back wall geometry $z_{1}, F_{i}\left(z^{1}{ }_{1}, z^{2}{ }_{1}, z^{3}{ }_{1}, \ldots, z^{j}{ }_{1}-0.01, \ldots, z^{16}{ }_{1}\right)$ denotes the temperature for the specimen with back wall geometry $\left(z^{1}{ }_{1}, z^{2}{ }_{1}, z^{3}{ }_{1}, \ldots, z^{j}{ }_{1}-0.01, \ldots, z^{16}{ }_{1}\right)$, e.g. with a $0.01 \mathrm{~cm}$ thinner back wall at the 4 th sampling point if $j=4$, see figure 5 (to the left). In reality, we are interested of a finer resolution of the thickness information. In figure 5 (to the right) and 6 , we see the analogue for sampling points in $0.1 \mathrm{~cm}$ distances, which was crucial for the good performance of the algorithm.
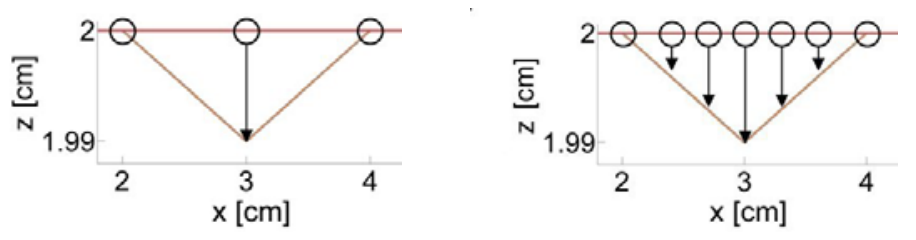

\footnotetext{
${ }^{1}$ The special case, finding the root of a real function, is known as Newton iteration and converges quadratically, if the initial value is "close enough" to zero.
} 
Figure 5: To the left: The initial geometry (red) was given for sampling points with $1 \mathrm{~cm}$ distances. The tiny change (yellow) of the initial geometry was applied for the sampling point at $x=3 \mathrm{~cm}$ width (to determine the 4th column of the derivative).

To the right as to the left, but the initial geometry was given for sampling points with $0.1 \mathrm{~cm}$ distances (and to determine the 31th column of the derivative). The arrows sketchily show the change of thickness at the neighbouring sampling points.

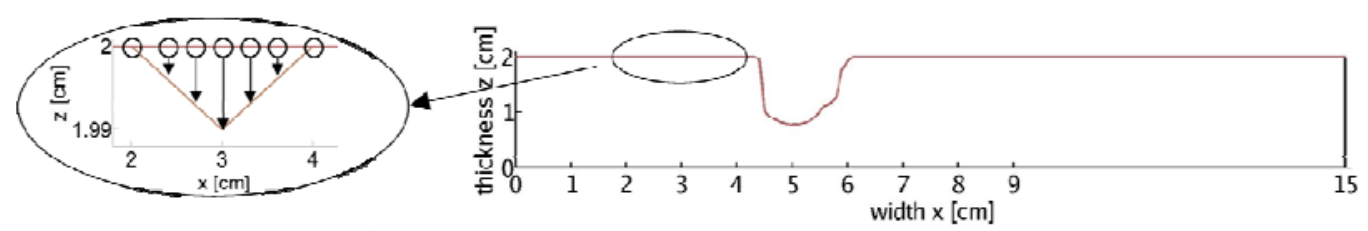

Figure 6. It shows figure 5 (to the right) from afar. Tiny change (yellow) of the initial geometry (red) at $x=3 \mathrm{~cm}$, given for sampling points with $0.1 \mathrm{~cm}$ distances.

The described difference method is time consuming: To calculate $F^{\prime}\left(z_{1}\right)$ for sampling points in $0.1 \mathrm{~cm}$ distances, we would need one simulation for $Z_{1}$ and 151 simulations for the geometries with tiny changes of $Z_{1}$. With the third a priori knowledge of the defect location within $x=[2,13] \mathrm{cm}$, we diminished the number of simulations. But the calculation of the derivative $F^{\prime}\left(z_{1}\right)$ is still time consuming, and we applied this method only in the 1 st and 21th iteration step.

To determine the derivative in the latter iteration steps, we chose the time efficient approximation using Broyden's method; for convergence research see [4]. Resulting from the secant equation, a known derivative at $Z_{k}$ defines the derivative at $Z_{k+1}$ in the following iteration step by the matrix equation Eq. (7)

$$
F^{\prime}\left(z_{k+1}\right)=F^{\prime}\left(z_{k}\right)+\left(\sum_{l=1}^{151}\left(z_{k+1}^{l}-z_{k}^{l}\right)^{2}\right)^{-1 / 2} \cdot\left[F\left(z_{k+1}\right)-F\left(z_{k}\right)-F^{\prime}\left(z_{k}\right) \cdot\left(z_{k+1}-z_{k}\right)\right] \cdot\left(z_{k+1}-z_{k}\right)^{T}
$$

where a superscript $T$ labels the transposed vector. As it requires no further simulation, Broyden's method delivered results after a few seconds instead of several hours.

For mitigating the disturbing impact of noise, the iterative Newton type method has to be regularised for this application. The Tikhonov regularisation [7] is well-known as a classical regularisation for its so-called mathematical optimality. As we considered an ill-posed problem and had noisy data, we modified the pseudo-inverted matrix

by

$$
F^{\prime}\left(z_{k}\right)^{+}=\left[F^{\prime}\left(z_{k}\right)^{T} \cdot F^{\prime}\left(z_{k}\right)\right]^{-1} \cdot F^{\prime}\left(z_{k}\right)^{T}
$$

$$
F^{\prime}\left(z_{k}\right)^{+} \approx\left[F^{\prime}\left(z_{k}\right)^{T} \cdot F^{\prime}\left(z_{k}\right)+\alpha \cdot I\right]^{-1} \cdot F^{\prime}\left(z_{k}\right)^{T}
$$

where $\alpha$ is a regularisation parameter adjusted to the signal-to-noise ratio, $I$ is the identity matrix, and a superscript $T$ labels the transposed matrix. We see: the larger we chose the parameter $\alpha$, the stronger the Tikhonov regularisation Eq. (9) deviates from the pseudo-inverted matrix Eq. (8). That means here, the geometry changes less and the inversion is immune to noise.

The iterative Newton type method Eq. (5) combined with Tikhonov regularisation (9) is known as LevenbergMarquardt method Eq. (10):

$$
z_{k+1}=z_{k}+\left[F^{\prime}\left(z_{k}\right)^{T} \cdot F^{\prime}\left(z_{k}\right)+\alpha \cdot I\right]^{-1} \cdot F^{\prime}\left(z_{k}\right)^{T} \cdot\left(T-F\left(z_{k}\right)\right)
$$

We optimised the regularisation to our application by choosing split regularisation parameters, depending on the sampling point (taking advantage of the second a priori knowledge). We chose a strong regularisation with a large parameter $\alpha=300$ for the sampling points where we wanted to preserve the initial value in thickness. For the other sampling points, we chose little regularisation with $\alpha=80$. Here, the considered time interval was limited to [10,600] s, due to unreliable simulation data in the first seconds. 


\section{Evaluation}

\subsection{Test specimen and experimental set-up}

We chose the material PVC, as we aimed a basic research: our first test phase of Levenberg-Marquardt on experimental data. PVC has an excellent emissivity and absorption coefficient of 0.96 without any surface treatment and a small thermal diffusivity (100 times smaller than the diffusivity of steel), which led to a slow thermal progress, and, thus, to a high signal-to-noise ratio by taking the average in time. We constructed two specimens with three flat bottom holes with remaining wall thicknesses of $0.15 \mathrm{~cm}, 0.3 \mathrm{~cm}$, and $0.6 \mathrm{~cm}$ and diameters of $1.2 \mathrm{~cm}$ and $0.8 \mathrm{~cm}$, respectively, see figure 7 .
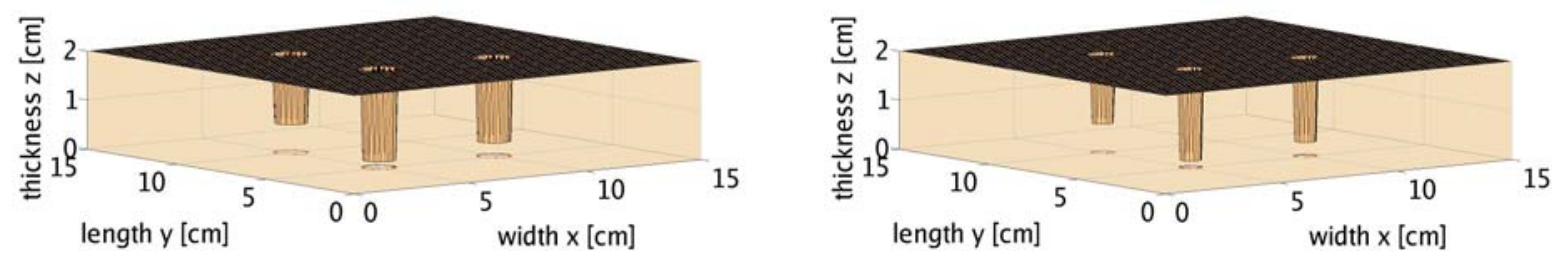

Figure 7: To the left: Specimen FBH12 with three flat bottom holes with diameter of $1.2 \mathrm{~cm}$ in a $2 \mathrm{~cm}$ thick, $15 \mathrm{~cm}$ wide and long PVC block. The remaining wall thicknesses are $0.15,0.3$ and $0.6 \mathrm{~cm}$.

To the right: Specimen FBH8 same as FBH12 with holes of $0.8 \mathrm{~cm}$ diameter.

We measured in reflection configuration: On the defect free front of the specimen, we temporarily heated up with two flash lamps (about $2.5 \mathrm{~ms}$ ) and measured the temperature distribution during and after heating as a function of time using an infrared camera. Here, an InSb camera was combined with a lens with a focal length of $10 \mathrm{~cm}$. The array size of the camera was $640 \times 512$, but only a frame size of about $475 \times 475$ pixels was analysed here. The frame rate was $93 \mathrm{~Hz}$; after averaging 93 frames, the frame rate resulted to $1 \mathrm{~Hz}$. Figure 8 shows the experimental set-up (to the left). For guaranteeing a more homogenous heating and for increasing the signal-to-noise ratio, we averaged 4 measurement cycles of the rotated PVC, such that, in the corresponding measurement cycle, the flash F2 provided a stronger heating on the right half, down half, left half and upper half of the PVC front. Figure 8 (in the middle and to the right) shows examples of the recorded temperature on the specimen $120 \mathrm{~s}$ after the flash, which displayed the best spatial contrasts; we subtracted a thermogram recorded before heating for minimising errors due to reflections on the specimen or an inhomogeneous initial temperature distribution.
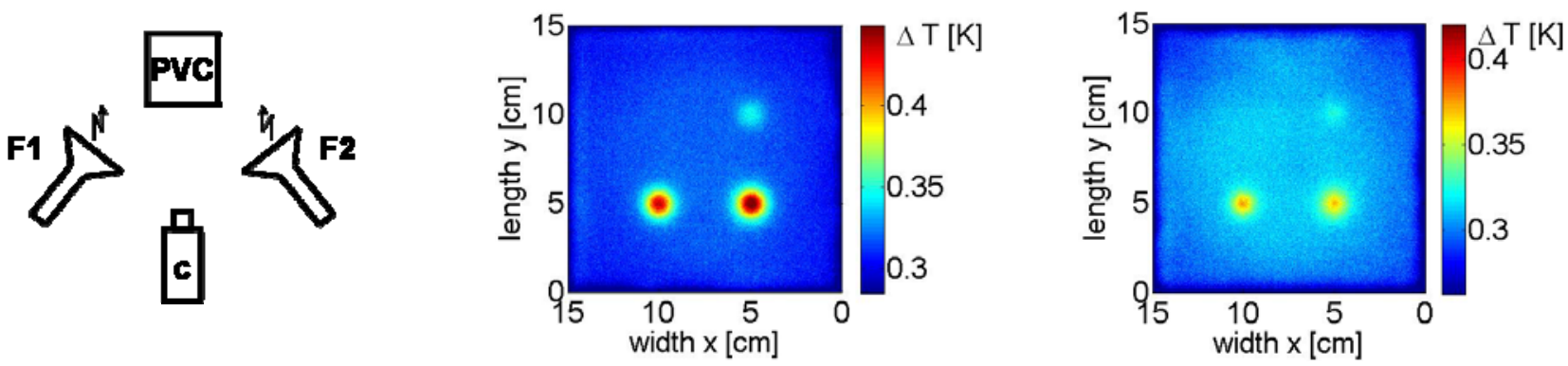

Figure 8: To the left: Sketch of the experimental set-up in reflection configuration. F1 and F2 are the flash lamps and c is the infrared camera.

In the middle: The thermogram of the heated FBH12 specimen, $120 \mathrm{~s}$ after the flash, showed the temperature difference in $K$ of the specimen related to room temperature (after averaging 4 measurement cycles, but before Gauss filtering).

To the right: The thermogram of the heated FBH8, $120 \mathrm{~s}$ after the flash, showed less contrasts as the holes had a smaller diameter. 

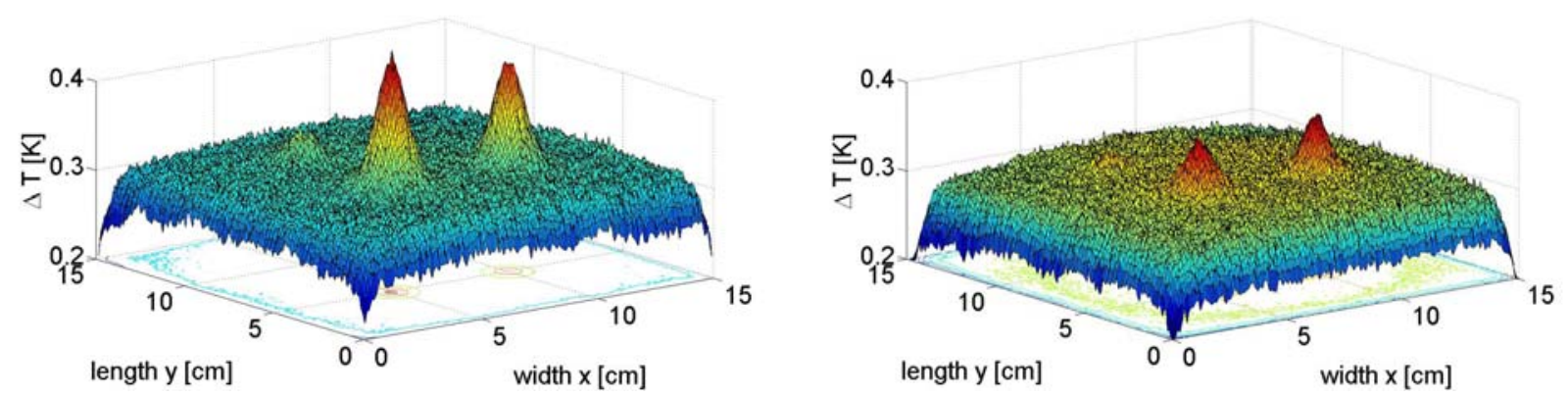

Figure 9: Another display of the data of figure 8, to compare the thermograms with the defect widths in figure 7. To the left: The thermogram of the heated FBH12 specimen, $120 \mathrm{~s}$ after the flash.

To the right: The thermogram of the heated FBH8, $120 \mathrm{~s}$ after the flash.

By applying a Gaussian lowpass filter in space and time, we increased the signal-to-noise ratio of the data. The Gaussian filter in space had the standard deviation $=40$ applied on 15 neighbouring values, and in time it had the standard deviation $=200$ applied on an increasing number of neighbouring values (increasing in the time interval from 30 to $110 \mathrm{~s}$ from 0 to 80 neighbouring values). By evaluating this spatial and temporal temperature distribution, we reconstructed quantitative information of the back wall geometry.

\subsection{Evaluation of the combination of the echo defect shape and the Levenberg-Marquardt method}

We evaluated the Levenberg-Marquardt method for its reconstruction performances. We chose supporting points with $0.1 \mathrm{~cm}$ distances, a requirement for a sufficient spatial resolution. Using the echo defect shape method Eq. (3), we got a first guess of the thickness, the initial geometry for the Levenberg-Marquardt method, see figure 10. As the required reference area, we chose a $3 \mathrm{~mm} \times 3 \mathrm{~mm}$ square at width $x=10 \mathrm{~cm}$ and length $y=10 \mathrm{~cm}$. All defect centres are located well and with the correct depth $z$, but the defect diameter is two times bigger than the real diameter. The defect-free area of FBH12 around the width $x=12 \mathrm{~cm}$ is assessed as flawed due to noise.
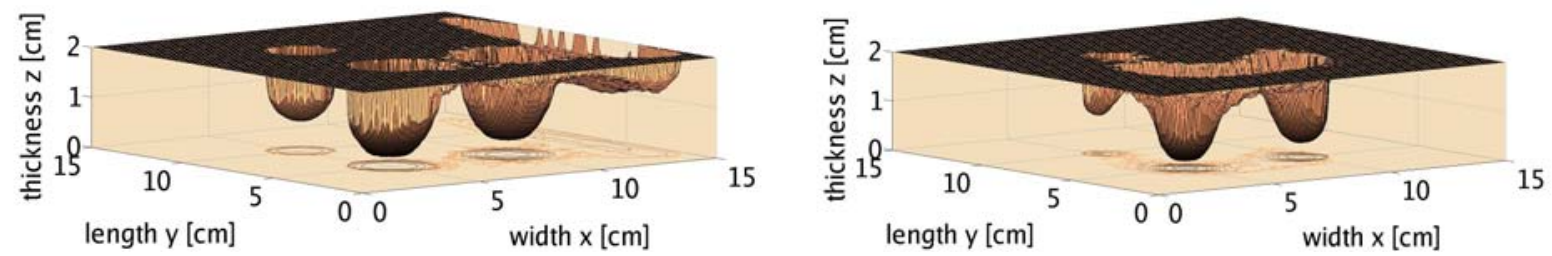

Figure 10: The initial geometry of specimen FBH12 (left) and FBH8 (right) before filtering it. All defects are located with the correct depth $\mathrm{z}$, the defect diameter is two times bigger than the real diameter.

The 3D initial geometry was smoothed with a Gaussian filter with standard deviation $=40$ applied on $2 \times 2$ neighbours, omitting the areas which we wanted to treat with care (see second a priori knowledge). By the LevenbergMarquardt method, we enhanced the two profiles at length $y=5 \mathrm{~cm}$ and $y=10 \mathrm{~cm}$, to reconstruct the two near-surface defects and the deep defect respectively. The $2 \mathrm{D}$ geometries in the $1^{\text {st }}$ and $21^{\text {st }}$ step of the Levenberg-Marquardt method were smoothed with a Gaussian filter with standard deviation $=40$ applied on 15 and 5 neighbours respectively, in order to calculate a meaningful difference quotient at sharp edges of the defect. By omitting the smoothing, a sharp edge would prevail, and we would reconstruct a thin fissure, additionally to the defect. The derivative was calculated by the difference quotient only at the $1^{\text {st }}$ and $21^{\text {st }}$ iteration step and approximated by the Broyden's method at the other steps. If we had used the difference quotient only for the $1^{\text {st }}$ step, the geometry enhancement would stagnate after about 20 steps, thus the reconstruction would be worse, especially at the reference area close to the defects. After 40 iteration steps, the reconstructed geometry was set as defect-free at all areas where the initial geometry (before the 2D smoothing) was defect-free, too.

To conclude, we see the real geometry, the initial geometry (before the 2D smoothing) using the echo defect shape method Eq. (3), and the resulting reconstructed geometry after 40 iterations using the Levenberg-Marquardt 
method in figures 11-14. Near-surface defects are well reconstructed, see figures 11 and 12, i.e. we did well reconstructions for the test specimen profiles at length $y=5 \mathrm{~cm}$.

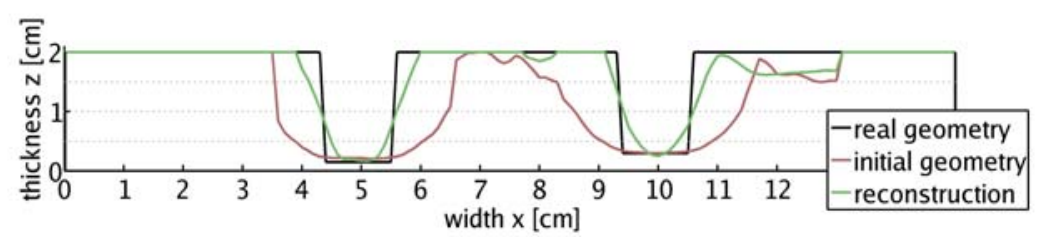

Figure 11: Specimen FBH12 profile at length $y=5 \mathrm{~cm}$; its real geometry, initial geometry and reconstruction in $2 D$.

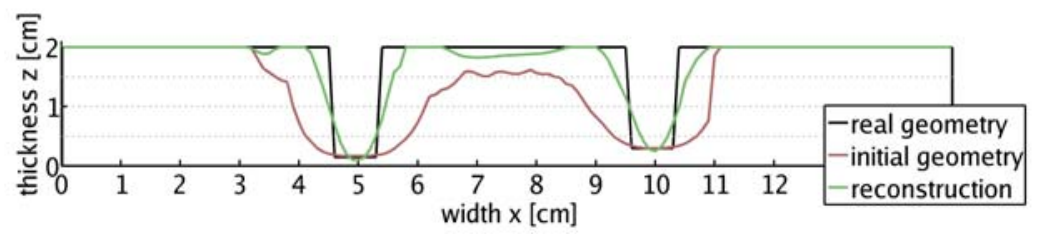

Figure 12: Specimen FBH8 profile at length $y=5 \mathrm{~cm}$; its real geometry, initial geometry and reconstruction in $2 D$.

Near-surface defect depths at $x=5$ and $10 \mathrm{~cm}$ are well reconstructed and differ in average only by $0.043 \mathrm{~cm}$, i.e. by taking the error Eq. (4) only for $i=51$ and 101. The reconstructed defect width is satisfying for $y=1 \mathrm{~cm}$, the initial width was more than double. The defect width, close to the surface, is too thin, and close to the back, it is too wide. We conclude, that sharp defect limitations are challenging.

In figures 13 and 14, we see the deep defects with a diameter of 1.2 and $0.8 \mathrm{~cm}$, respectively. Figure 13 shows an enhancement of the geometry in the defect widths, but a slightly worse defect depth.

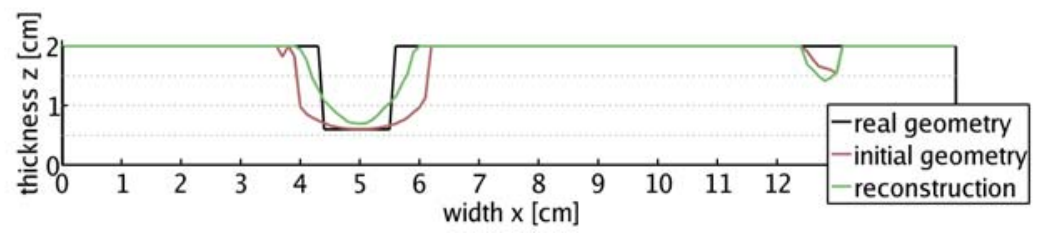

Figure 13: Specimen FBH12 profile at length $y=10 \mathrm{~cm}$; its real geometry, initial geometry and reconstruction in $2 D$.

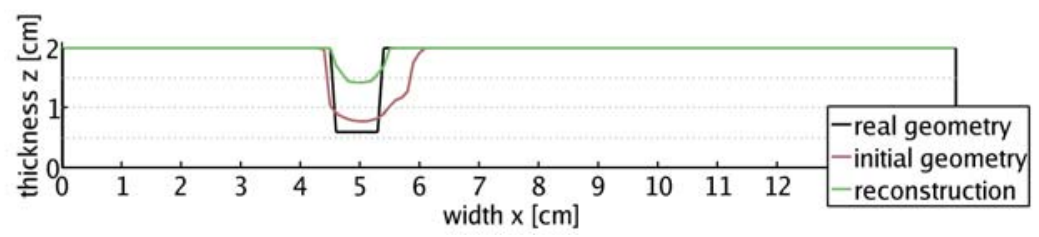

Figure 14: Specimen FBH8 profile at length $y=10 \mathrm{~cm}$; its real geometry, initial geometry and reconstruction in $2 D$.

The thinnest and deepest defect in figure 14 challenged the algorithm and showed the limit of the current state of the applicability. The initial geometry enhanced in the first 20 iteration steps (in the sense of error Eq. (4)), although the defect depth at $x=5 \mathrm{~cm}$ deteriorated. But the difference quotient in iteration step 20 forced a deteriorating depth profile using Eq. (4), apart from an even more deteriorating defect depth. To understand this undesired effect of the inversion, we discuss the belonging simulated temperature devolution.

The experimentally obtained temperature are shown in figure 15 , to the left; for $t=60,120$, and $240 \mathrm{~s}$ after the flash, at the front side of the FBH8 at length $y=10 \mathrm{~cm}$, filtered as described in chapter 3.1 and expressed as the difference to room temperature. Figure 15, in the middle and to the right, we show the simulated temperature for the real geometry and for the reconstruction, respectively. 

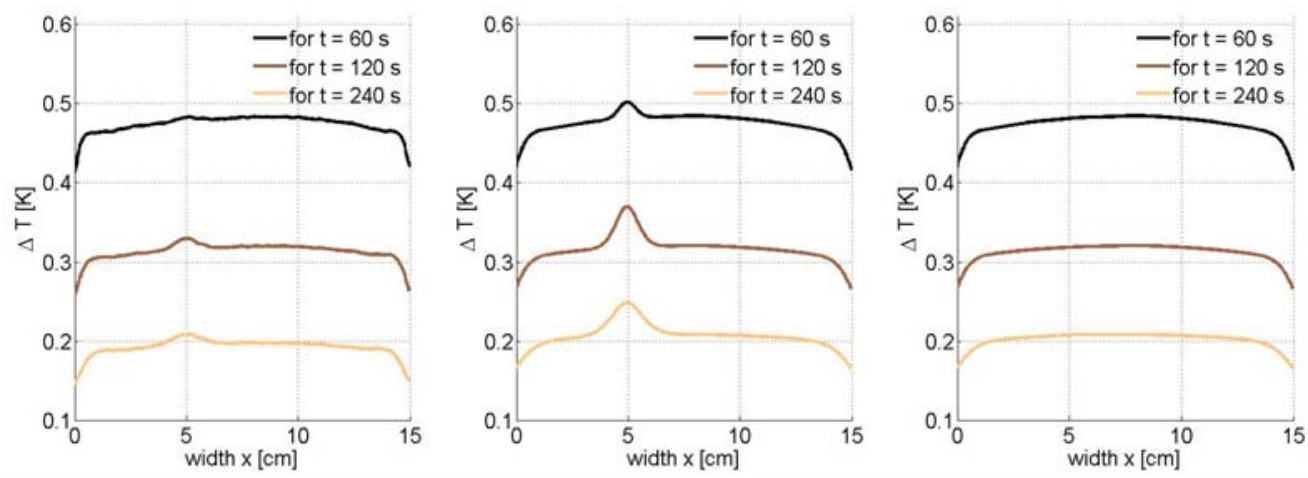

Figure 15: Temperature devolution of specimen FBH8 on a front line at length $y=10 \mathrm{~cm}$.

To the left: experimentally obtained temperature

In the middle: simulated temperature of the real geometry profile

To the right: simulated temperature of the reconstructed geometry of the

In the experiment, we see the scarcely visible spatial contrast of $0.015 \mathrm{~K}$ (to the left, brown line) - up to 200 times less than for the near-surface defects. In the simulation for the same geometry, we see spatial contrasts of $0.05 \mathrm{~K}$ (in the middle, brown line). Additionally, in the defect-free area, at $x=10 \mathrm{~cm}$, the simulated cooling is slower than in the experiment - the difference to the experiment increased up to $0.02 \mathrm{~K}$ with increasing time. This shows we have general discrepancies between simulation and experiment. In the simulation for the reconstructed geometry, we miss a visible spatial contrast (to the right, brown line).

We concluded that defects of this small size are sensitive to the discrepancies between our simulation and the experiment, such that it handicapped the reconstruction performance. One reason for the discrepancy is the theoretical disadvantage of the efficient 2D simulations, which neglect any heat loss in the missing length dimension. In our case, we neglected the lateral heat loss from the defect middle point at $x=5 \mathrm{~cm}$ to its surrounding in length dimension (i.e. $y=10 \pm 0$ ). This might have led to an excessively diminished defect volume.

\section{Conclusion and outlook}

We reconstructed back wall geometries of different PVC flat-bottom hole samples. The thickness of the 3D reconstruction by the echo defect shape method, was enhanced for 2D profiles using Levenberg-Marquardt method, an iterative inversion algorithm. The remaining wall thicknesses at the defect centres were well identified by the echo defect shape method. The Levenberg-Marquardt method enhanced especially the defect width, as it integrates lateral heat flow. The sharp defect geometries were challenging and led to the idea, to adjust the definition of derivation, such that even the reconstruction of defects with undercutting is a goal, in future time. For the reconstruction of very deep and small defects, like a flat-bottom hole with diameter of $0.8 \mathrm{~cm}$ and remaining wall thickness of $0.6 \mathrm{~cm}$, we suppose, that adjustments in the simulation model might lead to enhancements in the inversion procedure.

\section{References}

[1] Maldague X., "Nondestructive evaluation of materials by infrared thermography", Springer 1993

[2] Sun J. G., "Analysis of Pulsed Thermography Methods for Defect Depth Prediction", Journal of Heat Transfer, vol. 128, pp 329-338, 2006

[3] Omar M., Y. Zhou, "A quantitative review of three flash thermography processing routines", Infrared Physics \& Technology, vol. 51, pp 300-306, 2008

[4] Kaltenbacher B., Neubauer A., Scherzer O., "Iterative Regularization Methods for Nonlinear III-Posed Problems", de Gruyter, 2008

[5] Blome M., "Efficient measurement and data inversion strategies for large scale geoelectric surveys", Dissertation ETH 2009

[6] Lugin S., Netzelmann U., "A defect shape reconstruction algorithm for pulsed thermography", NDT\&E International, vol. 40, pp 220-228, 2007

[7] H. W. Engl, M. Hanke, A. Neubauer, "Regularization of Inverse Problems", Kluver, 1996 